\title{
REVISÃO: \\ Relação Entre o Comportamento de Aves, a Conformação da Paisagem Fragmentada e a Estrutura das Populações de Plantas
}

\author{
Bruno Senna Corrêa, Aloysio Souza de Moura
}

\section{RESUMO}

O processo de fragmentação do Bioma Cerrado é fruto da expansão agrícola. A paisagem fragmentada atualmente no Bioma é composta por manchas de fragmentos florestais isolados ou conectados por corredores de vegetação, natural ou introduzida. As aves são excelentes dispersores e contribuem para a conformação e dinâmica de ambientes fragmentados uma vez que estão diretamente relacionadas com os vegetais. São necessários estudos complementares e mais complexos envolvendo essa área, somando os esforços de estudos de frugivoria e dispersão, visando à reconstrução e reabilitação de ambientes fragmentados. No presente manuscrito são apresentadas algumas relações entre a distribuição e comportamento de aves e a estrutura da paisagem fragmentada, sob o ponto de vista da vegetação.

Palavras-chave: estrutura de paisagem, dispersão de propágulos, diversidade de aves

\section{Relation Between Bird Behavior, Landscape Conformation and Plant Population Structure}

\begin{abstract}
The process of spalling of the Cerrado Biome is fruit of the agricultural expansion. The fragmented landscape currently in this Biome is composed for spots of forest fragments isolated or hardwired by corridors of vegetation, natural or introduced. The birds are excellent dispersive and contribute for the conformation and fragmented environment dynamics a time that directly is related with vegetables. Complementary and more complex studies are necessary involving this area, adding the efforts of frugivory studies and dispersion, aiming at the fragmented environment reconstruction and whitewashing. In the gift manuscript some relations between the distribution and behavior of birds are presented and the structure of the broken up landscape, under the point of view of the vegetation.
\end{abstract}

Keywords: structure of landscape, dispersion of propagules, bird diversity

\section{INTRODUÇÃo}

Aves são excelentes representantes de fauna que agem como dispersores de sementes e propágulos. Nos ambientes tropicais, dentre os representantes da fauna, aves, insetos e morcegos são os principais dispersores dos vegetais. Desta forma, destaca-se sua importância na dinâmica de bordas em ambientes fragmentados e em conexões de paisagem fragmentada (corredores ecológicos).

O presente trabalho tem por objetivo: apresentar a importância de aves nos processos ecológicos de contribuição para a conformação de paisagem fragmentada e a estrutura das plantas nas fitofisionomias florestais (borda e interior).

\section{REFERENCIAL TEÓRICO}

\section{a) Comportamento das aves}

Os animais frugívoros exercem um importante papel na demografia das comunidades vegetais pois: a) interagem no momento em que as plantas estão no estágio final do ciclo reprodutivo, podendo favorecer ou comprometer o sucesso desta fase (Jordano, 1989); b) o padrão de deposição das sementes no ambiente pelos frugívoros afeta diretamente a sobrevivência das sementes e o estabelecimento das plântulas (Howe et al., 1985; Katusic-Malmborg e Willson, 1988), e c) promovem a distribuição espacial dos futuros indivíduos adultos na floresta ao descartar as sementes após a ingestão (Janzen et al., 1976; Jordano, 1992). Essas considerações são relevantes uma vez que, nas florestas tropicais, 50 a $90 \%$ das espécies arbóreas produzem frutos adaptados para a dispersão por animais (Howe e Smallwood, 1982). 
Em relação ao comportamento das aves, podemse relacionar os hábitos alimentares, com diferentes estratégias de forrageamento, os processos de nidificação diretamente relacionados à disponibilidade de recursos alimentares para permanência em determinada área, sendo este último, um fator diretamente relacionado com a distribuição da estrutura populacional de espécies vegetais de dispersão zoocórica. A estratégia reprodutiva é um dos principais fatores que levam as aves a buscar recursos energéticos para poder cumprir seu ciclo biológico. Para obter energia necessária aos processos vitais e à perpetuação da espécie, o processo evolutivo das aves permitiu o desenvolvimento de características específicas que incluem memória de localização, visão desenvolvida e estratégias complexas para forrageamento de recursos alimentares. Dessa forma, observa-se fundamental os processos de manuseio de frutos que visa, diretamente a busca por um recurso energético para a ave e, também diretamente uma estratégia pela qual a espécie vegetal poderá dispersar suas sementes. Esse processo de dispersão pode ser observado sob diferentes aspectos, tendo em vista a família ou as famílias de aves a serem atraídas, o tipo de dieta das famílias (guildas alimentares), se específico (somente frutos) ou generalista (frutos, insetos, néctar). Nesse aspecto, observam-se alguns aspectos relevantes para a situação de ambientes fragmentados, sejam eles naturais ou efeito de ação antrópica.

A comunidade vegetal de um determinado fragmento florestal está diretamente relacionada com a fauna dispersora. As espécies frutíferas apresentam um ciclo anual pré-definido pelo fotoperiodismo, ação dos fitohormônios, disponibilidade de luz, disponibilidade de água e recursos minerais no solo, relações ecológicas com outros organismos (micorrizas por exemplo são bastante comuns em raízes de espécies florestais), presença ou ausência de lâmina de água, entre outros. Esses fatores abióticos e os fatores bióticos estão diretamente relacionados com a dinâmica da comunidade vegetal e, portanto regem o comportamento das aves. Por exemplo, em casos de abertura de clareiras, ocorre alteração na dinâmica de dispersão de sementes devido às mudanças provocadas na estrutura horizontal e vertical da comunidade vegetal, podendo levar a novas taxas de recrutamento, podendo contribuir ou prejudicar a dinâmica de uma população. Krugel et al (2006) trabalhando com frugivoria de Nectandra megapotamica (Lauraceae) em floresta estacional decídua no Rio Grande do Sul. A espécie Nectandra megapotamica possui características que a incluem no sistema de dispersão generalista, exceto pelo alto valor nutritivo dos seus frutos. No presente trabalho, totalizando $70 \mathrm{~h}$ de observação focal registradas 726 visitas de 21 espécies de aves. As aves consideradas como potencialmente dispersoras de N. megapotamica foram Turdus albicollis, T. rufiventris, Pitangus sulphuratus e T. amaurochalinus entre as residentes, e
Tyrannus savanna e Myiodynastes maculatus entre as migratórias. As aves com dieta generalista pareceram favorecer a dispersão de N. megapotamica, pois consumiram os frutos inteiros, realizaram visitas curtas (menos de 3 minutos) e apresentaram maior freqüência de visitação que, por sua vez, está relacionada a uma maior remoção dos frutos.

A fragmentação e as bordas resultantes podem alterar a abundância de plantas frutíferas e aves frugívoras (Restrepo et al., 1999). Em relação ao processo de dispersão de frutos, observa-se que grande quantidade de espécies vegetais de florestas Neotropicais é dispersa por aves e mamíferos (Morellato e Leitão-Filho, 1992). Esse processo inclui espécies de árvores de dossel, com sementes grandes, dispersadas por frugívoros de maior porte (tucanos, jacus, cotingas, tinguaçus e planadeiras) (Howe e Smallwood, 1982), que estão propensos a se tornarem vulneráveis podendo fracassar na dispersão de sementes em ambientes fragmentados (Galetti, 2003). No caso de frutos pequenos de espécies de sub-bosque, uma ampla variedade de aves generalistas os consome (tangarás, sanhaços e tiranídeos) (Loiselle e Blake, 1999), sendo estas aves não consideradas altamente afetadas pela fragmentação (Aleixo e Vielliard, 1995).

Segundo Aizen e Feinsinger (1994) e Santos e Telleria (1994), é importante destacar o potencial de interrupção de interações mutualísticas entre animais e plantas, como polinização e dispersão de sementes. Algumas famílias de plantas como Melastomataceae e Rubiaceae, que produzem frutos escuros (pretos ou roxos) ou avermelhados são facilmente encontradas por aves de interior de florestas, que buscam recursos no subbosque. Essa guilda de plantas, apesar de ser muito importante para manutenção de aves frugívoras pequenas, parece não ser ameaçada pela modificação de hábitat em processos de fragmentação. (Silva et al., 2002).

Loiselle e Blacke (2002), sugerem que algumas espécies de aves podem ser muito importantes para determinadas guildas de plantas, como é observado com a extinção de Pipra sp., (Pipridae), pequena ave frugívora, extinta, na Costa Rica, que levou à redução drástica da chuva de sementes e da distribuição de algumas espécies de Melastomataceae.

Mudanças na composição das espécies de aves em fragmentos pequenos podem afetar a probabilidade de dispersão de sementes, podendo alterar a comunidade de sub-bosque (Galetti, 2003).

Wenny e Levey (1998) estudando a dispersão de sementes direcionada por em floretas tropicais chuvosas, observaram que quatro das cinco espécies de aves registradas jacu preto(Chamaepetes unicolor) Cracidae, quetzal (Pharomachrus mocinno) Trogonidae, tucano esmeralda (Aulacorhynchus prasinus) Ramphastidae, araponga (Procnias tricarunculata) Cotingidae, 
sabiá (Turdus plebejus) Turdidae, que geralmente permanecem na árvore em frutificação ou próxima a ela (Wheelwright, 1991) dispersaram a maioria das sementes dentro de $20 \mathrm{~m}$ da árvore parental em floresta com dossel fechado (fig 1 A). Em contraste, machos de aves canoras florestais, gastam em torno de $80 \%$ do dia vocalizando, onde advertem as fêmeas com altos sons (Snow, 1977). Tais cantos foram observados no estudo de Wenny e Levey (1998) próximos a árvores mortas, nas bordas de clareiras. Assim, a maioria das sementes dispersadas (59\%) por aves canoras em poleiros, caí ram em sítios mais distantes que 40 m das árvores conspecíficas e a maioria (52\%), também caiu em clareiras (Fig. 1 B). O teste de padrão total de dispersão obtido foi bimodal, com picos próximos às árvores parentais e sob o canto das aves canoras. A sobreposição entre os picos gerados pelas aves canoras e pelas outras espécies na dispersão foi pequeno (comparar A com B) e usualmente causado pelas fêmeas de aves canoras, que gastam pouco tempo nos poleiros e regurgitam sementes sobre ou perto das árvores parentais.
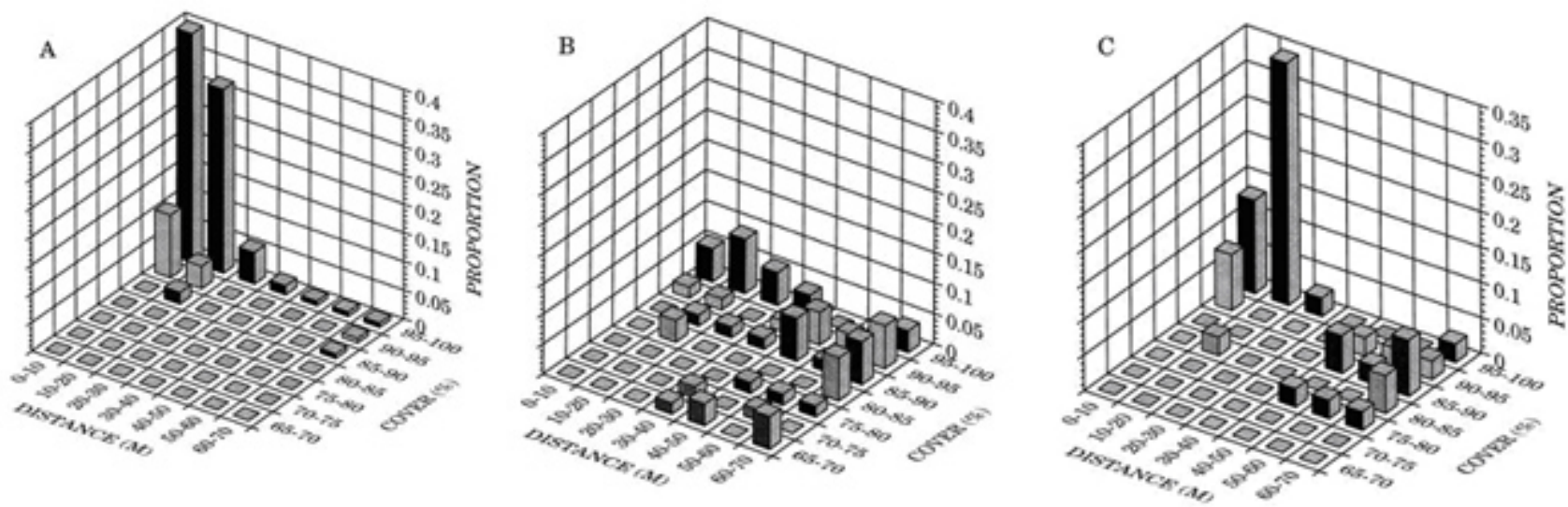

Figura 1. A frequência das sementes dispersadas ( $A$ e B ) e a sobrevivência de recrutas em 1 ano $(C)$ como funções da distância de $\mathrm{O}$. endresiana em frutificação mais próxima e a cobertura de dossel para jacus, quetzals, tucanos $(n=128)(A)$ e aves canoras $(n=56)(B)$. Cada barra representa a proporção de sementes dispersadas por dispersores particulares $(A$ e $B$ ) ou recrutamento $(C)$ em cada distância/categoria de cobertura de dossel. No estudo de Wenny e Levey, (1998) todas as aves canoras estavam mais distantes que $40 \mathrm{~m}$ da árvore frutífera mais próxima. Valores inferiores a $90 \%$ para cobertura de dossel representam clareiras. Notar a distribuição bimodal de recrutamento de indivíduos jovens $(\mathrm{C})$ que reflete a dispersão de semente, com um pico próximo às árvores parentais (A) e um segundo pico correspondente às aves canoras (B). O crescimento da entrada de sementes em grandes distâncias não é um resultado do crescimento de áreas com distância, mas é devido à concentração de sementes na vizinhança de poleiros de aves canoras. Em particular, o número de sementes dispersadas para clareiras por aves canoras foi maior que o esperado por tentativa $(X 2=36.45, \mathrm{df}=1, \mathrm{P}<0.001)$, baseado na área do sítio de estudo (5 ha) em clareiras (5,3\%) e floresta (94,7 \%). Não houve sobrevivência de recrutas nas duas categorias de dossel mais baixas, sugerindo um limite para o benefício de maiores níveis de luz (Wenny e Levey, 1998).

\section{b) Conformação da paisagem fragmentada (fragmentos, matriz, corredores)}

Os hábitats alterados na matriz circunvizinha de paisagem é uma fonte de colonizadores potenciais, também apropriados para colonização por espécies que podem persistir em tais hábitats. A habilidade de espécies para sobreviver em fragmentos pode depender dos hábitats circunvizinhos e se as espécies usam tais hábitats (Stouffer \& Bierregaard, 1995a,b; Laurance et al., 1997a,b; Renjifo, 2001). Em relação à tendência deste nível de paisagem para fragmentos florestais tropicais, observa-se maior atenção a valores de conservação de terras fora de reservas que incluem ambientes fragmentados, florestas secundárias, terras particulares e hábitats de campo como plantações de café (Brown \& Lugo, 1990; Turner \& Corlett, 1996; Daily, 2001)
Deve-se pensar em eventos que suplementam esforços de proteção de hábitats fragmentados, seja conservando hábitats selvagens contíguos a áreas protegidas, aumento nos níveis de conectividade dos fragmentos e restauração de áreas degradadas assim ganhando a terra como meios conservar a diversidade biológica (Laurance \& Bierregaard, 1997).

A natureza do remendo de paisagens assegura que populações de muitas espécies de plantas estejam espacialmente isoladas umas das outras, por $100 \mathrm{~m}$ ou mais. Para cada espécie, a dispersão de sementes representa a única maneira que as populações podem trocar indivíduos ou colonizar ambientes vazios, mas apropriados (Cain et al., 2000).

A dispersão das sementes é um processo demográfico chave na vida das plantas por representar a 
ponte que une a polinização com o recrutamento que levará ao estabelecimento de novas plantas adultas (Harper, 1977). A fecundidade de uma planta em particular ou de uma população de plantas depende não somente do sucesso no estágio de polinização, mas também no sucesso de estabelecimento e crescimento dos novos indivíduos. As novas sementes produzidas a cada estação reprodutiva representam não apenas novos indivíduos que são acrescidos à população em termos numéricos, mas também distintos genótipos a serem acrescentados ao acervo genético populacional. Portanto, a dispersão das sementes une todo o ciclo reprodutivo das plantas e pode ter importantes conseqüências para a demografia e a estrutura genética populacionais (Jordano \& Godoy, 2002).

O grau de isolamento do fragmento, isto é, uma medida de quão separado o fragmento está de outras áreas que podem servir como fonte de animais e sementes, é um fator importante para as interações entre as plantas e os dispersores de sementes. Muitos animais frugívoros não atravessam áreas abertas ou evitam ambientes perturbados (Estrada et al., 1993; Silva et al., 1996). Dessa forma, fragmentos isolados tendem a receber menos sementes de outras áreas e apresentar menor abundância e riqueza de animais frugívoros. A literatura mostra que o grau de isolamento de um fragmento está negativamente relacionado com a riqueza de plantas zoocóricas (van Ruremonde \& Kalkhoven,1991; Ochoa-Gaona et al., 2004). Os efeitos do isolamento podem ser minimizados pela composição da matriz que circunda o fragmento e, em maior escala, pela composição da paisagem onde o fragmento está inserido. Esses dois fatores podem influenciar a conectividade do ambiente e, portanto, o fluxo de animais e sementes no fragmento (Metzger, 2000; Graham, 2001). A permeabilidade da matriz à fauna, é um fator relevante na dinâmica da comunidade vegetal. Em fragmentos florestais amazônicos, por exemplo, matrizes compostas por árvores do gênero Cecropia (Cecropiaceae) são mais favoráveis à fauna que matrizes onde predominam plantas do gênero Vismia (Clusiaceae) e, em comparação com pastagens, ambos os tipos de matrizes são mais permeáveis à fauna (Laurance et al., 2002). A influência da paisagem na composição faunística de um fragmento pode se estender por vários quilômetros. Na Austrália, Price et al. (1999) verificaram que a porcentagem de cobertura florestal em um raio de $50 \mathrm{~km}$ a partir de determinado fragmento de floresta foi determinante para a ocorrência de algumas espécies de aves frugívoras nos fragmentos. No sudeste do Brasil, aves migratórias são comuns em fragmentos de floresta onde participam ativamente da dispersão das sementes (Galetti \& Pizo, 1996; Pizo, 1997). Naturalmente, a ocorrência destas aves nos fragmentos e, conseqüentemente, o seu papel como dispersoras de sementes sofrem influência das alterações ambientais que ocorrem ao longo da rota de migração, que pode se estender por várias centenas de quilômetros (Martin \& Karr, 1986). Concluindo, as interações entre plantas e dispersores de sementes estão sujeitas a uma série de alterações em ambientes fragmentados. A magnitude dos efeitos da fragmentação sobre estas interações depende de: (1) fatores que, em escalas local e regional, afetam a dinâmica das populações de animais frugívoros em fragmentos, alterando sua composição e abundância, (2) características das plantas que as tornam mais ou menos suscetíveis à extinção ou diminuição na abundância dos dispersores e (3) características inerentes à interação. Dentre os primeiros destacam-se a área, a forma e o grau de isolamento do fragmento, as perturbações a que está ou esteve sujeito (por exemplo, extração de madeira, caça, fogo), a natureza da matriz circundante e a conectividade da paisagem onde se insere. Características importantes da planta são a morfologia do fruto e suas exigências para germinação das sementes e estabelecimento das plântulas (Bruna, 1999; Benítez-Malvido \& Martínez-Ramos, 2003). Em relação às características da interação em si, destacam-se o grau de especialização da interação e o nível de redundância no papel desempenhado pelos vários dispersores que participam da interação. Interações mais especializadas, ou seja, que envolvem menor número de participantes, são naturalmente mais suscetíveis à extinção ou diminuição na abundância dos dispersores (Asquith et al., 1999). A maioria dos sistemas de dispersão, no entanto, envolve vários dispersores e é possível que alguns deles sejam funcionalmente redundantes no que se refere ao papel que desempenham como dispersores de sementes. As evidências disponíveis, entretanto, mostram que isso raramente ocorre, pois os diferentes dispersores diferem em aspectos qualitativos e/ou quantitativos da dispersão que promovem e a extinção de um único dispersor, ou pior, de um conjunto deles, pode resultar em alterações importantes para a estrutura e o recrutamento das populações de plantas (Loiselle \& Blake, 2000) e, em última instância, para toda a comunidade (Howe, 1984). Assim, se entendemos que a biodiversidade vai além do conjunto de espécies que compõem o ambiente e engloba também as interações entre elas, justifica-se plenamente todo o esforço de manejo dos fragmentos e da paisagem circundante para minimizar os efeitos da fragmentação sobre as interações entre as plantas e os dispersores de suas sementes.

A principal influência demográfica da dispersão das sementes advém de processos de limitação associados ao número limitado de sementes que são dispersas com sucesso ou à limitada chegada de sementes a locais que oferecem alguma possibilidade de recrutamento bem-sucedido. Os efeitos dos animais frugívoros sobre as plantas vão além da remoção das sementes, pois eles têm múltiplas, e nem sempre imediatas, influências sobre as sementes, plântulas e jovens. Os animais frugívoros podem limitar o crescimento 
populacional das plantas se a quantidade de sementes que dispersam é insuficiente ou se a qualidade de dispersão que promovem é inadequada (isto é quando depositam sementes em locais com baixa probabilidade de recrutamento; (Schupp, 1993). A idéia de limitação no recrutamento das plantas relacionada à atividade dos animais diz que esta atividade é insuficiente para produzir o máximo recrutamento a partir das sementes produzidas em uma dada estação reprodutiva; se a atividade dos animais frugívoros fosse aumentada, teríamos um maior número de sementes removidas para locais seguros para o recrutamento. Por exemplo, os frugívoros podem dispersar com sucesso uma alta proporção das sementes produzidas em uma população de plantas. No entanto, eles limitam o recrutamento nesta população se a dispersão é direcionada para locais de alta densidade coespecífica, onde prevalecem mecanismos de mortalidade dependentes de densidade, ou seja, se depositam as sementes em locais de baixa qualidade (Jordano et al., 2006).

Uma revisão das limitações inerentes à sucessão florestal em áreas degradadas tropicais a partir da chuva de sementes produzida por frugívoros é apresentada por Duncan \& Chapman (2002), dentre as quais podemos destacar as seguintes:

a) Condições inóspitas de temperatura, umidade e nutrientes no solo que irá receber as sementes, dificultando a germinação e o estabelecimento.

b) Altas taxas de predação, principalmente por roedores e formigas.

c) Competição local com gramíneas de rápido crescimento, que limitam o desenvolvimento das plântulas de espécies zoocóricas.

d) Ocorrência freqüente do fogo em paisagens abertas e degradadas.

e) Baixo número de sementes que chegam às áreas alteradas, em quantidade insuficiente para dar início a um processo sucessional bem-sucedido.

f) Chuva de sementes nem sempre é dirigida aos sítios onde o estabelecimento de espécies arbóreas é esperado.

g) Na composição específica da chuva de sementes gerada por frugívoros em áreas degradadas, geralmente faltam espécies arbóreas dos estágios intermediários e avançados da sucessão, cujo recrutamento é necessário para alavancar o processo sucessional para além do estágio de pioneiras.

h) O recrutamento de espécies lenhosas a partir de poleiros artificiais em áreas muito grandes deveria exigir alta densidade dessas estruturas, elevando os custos da sua implantação e diluindo a chuva de sementes entre os poleiros.

Há ainda quatro conceitos-chave no processo de limitação que são derivados do estágio de dispersão das sementes: limitação na produção de fruto (ou limitação de fonte; Clark et al., 1999; Turnbull et al., 2000), limitação de dispersão, limitação de recrutamento e limitação de estabelecimento (Jordano \& Godoy, 2002; Muller-Landau et al., 2002). Os fatores que influenciam o número absoluto de sementes disponível para a dispersão são determinados durante a fase pré-dispersão, de maneira que o número total de sementes produzidas em uma população representa o número máximo de sementes que pode efetivamente recrutar em um dado episódio reprodutivo. Se a atividade dos animais frugívoros não é limitante, todas essas sementes poderiam eventualmente recrutar. Polinização ineficiente, conflitos de alocação de recursos, predação de flores e predação pré-dispersão das sementes estão entre as principais causas da limitação de fonte. A limitação de recrutamento abrange todos os estágios entre a remoção dos frutos e o estabelecimento dos adultos nos quais pode haver a perda de propágulos (Muller-Landau et al., 2002), embora ela seja mais freqüentemente aplicada aos estágios pós-dispersão, isto é, após a deposição das sementes pelos dispersores. A limitação da dispersão inclui todos os processos que podem limitar o número de sementes que chegam a locais seguros para o recrutamento; portanto, ela engloba a remoção e a deposição das sementes. Se as sementes são dispersas em quantidade suficiente, porém são depositadas em locais de baixa qualidade, então o recrutamento é limitado pela atividade dos dispersores, isto é, o estabelecimento é limitado pela dispersão de má qualidade e não pela eventual pequena quantidade de sementes dispersadas. Portanto, podemos entender a limitação de dispersão como potencialmente agindo durante duas fases: na limitação de dispersão em si e na limitação de estabelecimento. Junto com a limitação de fonte, a limitação de dispersão é o principal fator a determinar potencialmente a limitação de recrutamento, isto é, o insucesso da prole produzida em um dado episódio reprodutivo em se estabelecer produzindo adultos reprodutivos. Isto representa uma visão expandida da dispersão como um estágio-chave no recrutamento das plantas, que enfatiza o papel potencial das interações com os dispersores de sementes sobre os efeitos que afetam as populações de plantas (Jordano et al., 2006).

Um protocolo para o estudo da limitação de recrutamento foi recentemente proposto por Muller-Landau et al. (2002). Começando pelo monitoramento da chuva de sementes por meio de coletores de sementes é possível monitorar a emergência de plântulas associadas a esta chuva de sementes em diferentes pontos de determinada área. A limitação na chegada de sementes (limitação de fonte) pode ser estimada como a proporção de pontos de amostragem que não receberam sementes. A limitação de dispersão pode ser estimada comparando-se a proporção de coletores que de fato receberam 
sementes com à proporção que seria esperada segundo um modelo randômico de dispersão das sementes. Por fim, a limitação de estabelecimento pode ser estimada combinando-se os dados de chegada das sementes e emergência das plântulas nos pontos de amostragem e calculando-se em seguida a redução no estabelecimento de plântulas nos pontos onde as sementes chegaram (Muller-Landau et al., 2002). Estes métodos para estimar os processos de limitação basicamente dividem o número de oportunidades de recrutamento perdidas (ou seja, locais onde a espécie não recruta) entre aquelas perdidas devido à não chegada das sementes e aquelas perdidas devido a falhas no estabelecimento. Estes estudos nos informam que a maioria (isto é, > $>00 \%$ ) das espécies arbóreas tropicais em uma dada localidade atinge com suas sementes não mais que, em média, $5 \%$ dos coletores de sementes por ano, o que sugere extensa limitação de fonte. Tal limitação pode ser ainda mais exacerbada caso os dispersores tenham, por ação antrópica, desaparecido de determinada área (Jordano et al., 2006).

Estudos da estrutura da comunidade de aves paisagens fragmentadas (fragmentos florestais neotropicais e plantações de café e cardamom (tempero)) na Índia, relacionando o efeito do estrato vegetal, a conectividade e a distribuição de aves de floresta. O total de espécies de aves observadas para os 4 estratos de hábitat foi: 43 espécies (controle - floresta tropical chuvosa - 1 sítio), 95 espécies (fragmentos de floresta tropical chuvosa - 5 sítios), 76 espécies (plantações de café -5 sítios) e 49 espécies (plantação de cardamom - 1 sítio). Entre o total de espécies na comunidade em cada um dos 4 estratos (controle, fragmentos, plantação de café e plantação de cardamom) a porcentagem de espécies de aves de floresta tropical chuvosa foi maior no controle $(95,3 \%)$ e na plantação de cardamom sob sombra natural $(89,9 \%)$. Aves de floresta secundária infiltraram dentro de fragmentos de floresta tropical chuvosa e em plantações de café e a porcentagem de espécies de aves de floresta tropical chuvosa foi menor $70,5 \%$ e $59,2 \%$, respectivamente para cada estrato. Essas diferenças no número de espécies de floresta primária e floresta secundária entre os 4 estratos trabalhados foi estatisticamente significante $X^{2}=34.1$, df $=3, P<0.001$ (Raman, 2004).

No caso da relação de corredores ecológicos e aves dispersoras de sementes, observa-se ser de grande importância à presença de aves frugívoras presentes em ambientes de corredores. Os corredores podem ser artificiais ou naturais e, tem por objetivos, permitir a movimentação de espécies chave da fauna (endêmicas, raras, ameaçadas), o fluxo gênico de espécies vegetais (preferencialmente dispersadas pela fauna), fornecer recursos para a fauna e permitir o uso desses recursos para possibilitar a ocorrência dos processos ecológicos necessários para a história ecológica do ambiente. Den- tre os representantes de fauna silvestre em ambientes fragmentados, os maiores dispersores são as aves. Dentre os critérios para avaliação dos usuários potenciais nos corredores observa-se: 1. o status de distribuição das espécies; 2 . o grau de dependência de hábitats florestais; 3 . o grau de mobilidade através de áreas abertas; 4 . o grau de especialização de hábitat; 5 . a resposta das espécies ao desmatamento (sensível, não sensível); 6. o status de conservação das espécies. Entretanto, devese levar em consideração que existe uma relação direta entre fauna e flora e este fator é fundamental para o sucesso reprodutivo das espécies e manutenção do equilíbrio dinâmico destes ambientes. Fatores como o design, a largura, a composição de espécies vegetais, a distância, a influência da matriz circunvizinha e as conseqüências dessa matriz na estrutura dos corredores são essenciais para a avaliação dos processos ecológicos. O design dos corredores artificiais criados visando o deslocamento de fauna geralmente é planejado através de fotografias aéreas e tendem a ser lineares. Esse formato visa facilitar o deslocamento dos organismos. A largura deve ser mensurada em conjunto com outros fatores como composição de espécies vegetais e matriz circunvizinha. Corredores muito largos $(50 \mathrm{~m}-100 \mathrm{~m})$, podem sofrer efeitos de fatores como fogo, construção de estradas, efeito borda, gerando alterações na dinâmica da comunidade do corredor e dos fragmentos conectados, declinando assim sua eficiência ao longo dos anos (Saunders e Hobbs, 1991). Alterações ocorrentes na matriz, irão gerar efeito borda; se o corredor for muito estreito $(10 \mathrm{~m})$, a intensidade do efeito borda na borda e no interior do fragmento serão bastante parecidos. A estratificação vertical e a distribuição horizontal de espécies vegetais presentes na área do ambiente fragmentado, irão auxiliar na eficiência da dispersão de sementes pela fauna (principalmente avifauna). Observase a importância de aves na dispersão de sementes em estudo realizado em fragmentos florestais conectados por corredores ecológicos no município de Lavras, MG, no Cerrado. A área de estudo compreende uma matriz de plantações de café, milho, soja e cana-de-açúcar e fragmentos floretais de floresta estacional conectados por corredores ecológicos, plantados em meados do século passado que serviam como divisores de terra. A atual estrutura dos corredores compreende na presença de espécies vegetais presentes nos fragmentos florestais da região, largura em torno de 3 a $4 \mathrm{~m}$ e comprimento que varia de $300 \mathrm{~m}$ a $2 \mathrm{~km}$. É notável o deslocamento de aves dispersando frutos através dos corredores. Tanto espécies generalistas como Pitangus sulphuratus, Myiozetetes similis (Tyrannidae) e Furnarius rufus (Furnariidae) como espécies especialistas como Chiroxiphia caudata (Pipridae), Pachyramphus castaneus (Cotingidae), Vireo olivaceus (Vireonidae), Basileuterus flaveolus (Parulidae) e Mackenziana leachii (Thamnophilidae), podem ser observadas ao longo dos corredores em diferentes 
horários do dia. Esses dados sugerem que a capacidade de adaptação das aves é a resposta ao nível de isolamento e à qualidade do hábitat na busca de recursos. $\mathrm{A}$ eficiência dos corredores estudados é parcial em pelo menos dois aspectos: 1 . se por um lado observa-se a tendência de sucesso reprodutivo de espécies vegetais florestais, seja pelo local de deposição das sementes pelas aves, como pelo fluxo gênico que pode ocorrer em fragmentos ou corredores vizinhos; 2. no conceito de metapopulações, a dinâmica das comunidades em cada fragmento podem sofrer influência de diferente fatores em diferentes intensidades; dessa forma, pode-se pensar em diferentes situações de dinâmica dentro de cada fragmento, que ao ser visitado por grupos de dispersores, podem receber recursos biológicos que pode favorecer ou dificultar os processos ecológicos, podendo levar ao declínio populacional de espécies vegetais florestais de interior, reduzindo assim a diversidade de espécies de um fragmento. Parece que a matriz circunvizinha influencia diretamente a distribuição de aves ao longo da matriz e quem sabe pode influenciar a dinâmica da comunidade vegetal nos fragmentos e corredores. Para descobrir esse aspecto, é necessário relacionar, dentre outros fatores, a relação das espécies de aves frugívoras e espécies vegetais por ela dispersadas, em corredores, em fragmentos e na matriz (Corrêa et al., 2011 no prelo).

Para permitir a correta implantação e utilização eficiente de corredores ecológico é necessário implementar estratégias de manejo que incluam: 1 . coleta de dados quantitativos de movimentação de organismos em corredores (escala, taxas e freqüência de movimentos); 2.coleta de dados de populações animais que vivem em corredores; 3 . coleta de dados de ecologia e comportamento de organismos que vivem em corredores e como isso afeta o uso dos corredores (informações sobre estratégias de sobrevivência, uso de recursos, capacidade de deslocamento).

\section{c) Estrutura das populações de plantas}

Teorias de dispersão de sementes e regeneração de florestas tropicais sugerem que as vantagens da dispersão para a maioria das plantas é escape da predação próxima à árvore parental e colonização de locais vagos (clareiras), locais nos quais são imprevisíveis no espaço e no tempo. Aves possuem um papel significativo na dispersão de sementes fornecendo dispersão direta a sítios favoráveis e, portanto podem influenciar nos padrões de recrutamento e na diversidade de espécies em florestas tropicais (Wenny e Levey, 1998).

A dispersão de sementes determina o arranjo especial e o ambiente físico das sementes, dessa forma é um importante passo para o ciclo reprodutivo da maioria das plantas (Harper, 1977; Willson, 1992). Em florestas Neotropicais, aves são importantes, porque acima de $75 \%$ das espécies de plantas produzem frutos ingeridos por aves (Stiles, 1985; Herrera et al., 1994). A fragmentação de florestas é um processo que reduz o ambiente a parcelas menores. Esse processo leva ao desaparecimento de espécies de aves florestais de interior e, conseqüentemente promove alterações na dinâmica de espécies vegetais com frutos dispersados por ornitocoria (Cordeiro \& Howe, 2003).

Aves que dispersam sementes de Leptonychia (Sterculiaceae) em florestas contínuas são raras ou ausentes em pequenos fragmentos, sendo observado menor quantidade de sementes removidas de cada árvore e menor indivíduos jovens de Leptonychia em fragmentos menores do que em florestas contínuas. Esse resultado forneceu evidência sólida da deficiência da dispersão em ambientes fragmentados e os impactos no ciclo reprodutivo de espécie vegetal dispersada por aves (Cordeiro \& Howe, 2003).

Considerando os custos energéticos da planta para produção de estruturas de dispersão, tipo pericarpo carnoso, como recompense, é razoável esperar vantagens na dispersão de sementes (Thompson \& Willson, 1978; Wheelwright \& Orians, 1982). Três vantagens não exclusivas foram propostas por Howe $(1982,1986)$ : (i) escape de alta mortalidade de sementes ou recrutas sob e perto de árvores parentais (Hipótese de Escape); (ii) colonização de sítios imprevisíveis, efêmeros ou novos (clareiras) (Hipótese de colonização); e (iii) dispersão direta a microhábitat favoráveis (Hipótese de dispersão direta).

A hipótese de Escape (Janzen, 1970; Connell, 1970) é esperada ser uma vantagem para a maioria das plantas e é baseada em numerosos estudos de densidade ou distância dependentes da mortalidade próximo aos parentais (Howe, 1982; Howe et al., 1985; Clark \& Clark, 1984; Crawley, 1992; Wilson, 1992; Wills et al., 1997). Entretanto, a colonização ou dispersão direta também pode ser importante para que sementes escapem da mortalidade. A hipótese da colonização é mais relevante quando sítios apropriados para o estabelecimento são imprevisíveis ou aleatoriamente distribuídos, com é o caso de abertura de clareiras pela queda de árvores em florestas tropicais (Howe, 1982; Murray, 1988; Van der Meer \& Bongers, 1996). Em florestas tropicais chuvosas, praticamente todo o dossel de espécies de plantas (não obstante o grau de tolerância à sombra) necessita de um mínimo de clareiras para alcançar a maturidade reprodutiva (Hubbel a Foster, 1986; Swaine \& Whitmore, 1988; Denslow \& Hartshorne, 1994; Clark, 1994). A estratégia esperada de dispersão dessas espécies de plantas é via colonização, cobrindo o solo com propágulos capazes de realizar dormência ou crescimento suprimido até a formação de clareiras e o aumento dos níveis de luz que permitam a germinação, crescimento rápido e/ou maiores chances de sobrevivência (Howe, 1982; Brandani et al., 1988; Schupp \& Howe, 1989). Alternativamente, a 
dispersão direta pode resultar se a planta atrai dispersores que depositam sementes não aleatoriamente em locais apropriados, desse modo aumentando a aptidão da planta (Reid, 1989; Sargent, 1995). Embora a dispersão direta tem sido postulada para dispersão por vertebrados em plantas tropicais (McKey, 1975; Howe, 1982; Levey, 1988) nunca foi demonstrado através de dispersão não aleatória para sítios favoráveis para crescimento de recrutas e sobrevivência.

A relação entre aves canoras e a estrutura populacional de plantas em ambientes fragmentados, entre outros fatores, está relacionado aos diferentes tipos de frutos ingeridos pelas espécies de aves dispersoras (Wheelwright et al., 1984), estando ativas em poleiros por pelo menos 4 meses em ambientes florestais $\mathrm{Ne}-$ otropicais durante a estação reprodutiva (Snow, 1977). $\mathrm{O}$ processo de dispersão, associado à diversidade de espécies vegetais frutíferas e os fatores abióticos, irão propiciar a dinâmica das populações vegetais no fragmento, favorecendo uma heterogeneidade espacial e vertical diretamente relacionada com esses fatores. Corroborando os resultados observados por Wenny \& Levey (1998), o padrão de dispersão de frutos para espécies comuns de plantas por aves canoras é bastante parecido ao observado em Monte Verde, assim como em florestas Neotropicais onde existem aves canoras. Outras espécies que usam habitualmente poleiros ou sítios de defecação como dançarinos (Pipridae) podem ter influência desproporcional no local de recrutamento de espécies de plantas (Krijger, 1997).

A dispersão de sementes de Ocotea endresiana por aves canoras é uma das ligações mais evidentes entre a dispersão e o sucesso reprodutivo de árvores florestais tropicais já documentado (Wenny \& Levey, 1998). A falta de outros exemplos similares vão da dificuldade de se observar os dispersores através do dossel até a deposição de sementes. A dinâmica de clareiras é importante e necessária para regeneração de muitas espécies de árvores e dispersores como as aves canoras parecem promover dispersão direcionada para clareiras. A perda desses dispersores pode levar a uma redução da aptidão para espécies de árvores levando a alterações na estrutura da comunidade de florestas tropicais.

\section{CONCLUSÃO}

São necessários estudos complementares e periódicos (décadas de banco de dados) da dinâmica de aves em ambientes de borda de fragmentos florestais nas mesmas áreas, como estratégias de predição da conformação das paisagens em áreas fragmentadas. $O$ conhecimento de alguns destes atributos ecológicos podem direcionar estratégias específicas para projetos de preservação de áreas prioritárias (hotspots).

\section{REFERÊNCIAS BIBLIOGRÁFICAS}

AIZEN, M.A.; FEINSINGER, P. Forest fragmentation, pollination, and plant reproduction in Chaco dry Forest, Argentina. Ecology, Durham, v. 75, p. 330-351, 1994.

ALEIXO, A.; VIELLIARD, J. M. E. Composição e dinâmica da avifauna da mata de Santa Genebra, Campinas, São Paulo, Brasil. Revista Brasileira de Zoologia, Viçosa, v. 12, n. 3, p. 493-511, 1995.

ASQUITH, N. M., J. TERBORGH, A. E. ARNOLD \& M. RIVEROS. The fruits the agouti ate: Hymenaea courbaril seed fate when its disperser is absent. Journal of Tropical Ecology, New York, v. 15, p. 229-235, 1999.

BENITEZ-MALVIDO, J. \& M. MARTÍNEZ-RAMOS. Impact of forest fragmentation on understory plant species richness in Amazonia. Conservation Biology, Cambridge, v. 17, p. 389-400, 2003.

BRANDANI, A.; HARTSHORN, G.S.; ORIANS, G. H. Journal of Tropical Ecology, New York, n. 4, p. 99119, 1988.

BROWN, S.; LUGO, A. E. Tropical secondary forests. Journal of Tropical Ecology, New York, v. 6, n. 1, p. 1-32, 1990.

BRUNA, E. M. Seed germination in rainforest fragments. Nature, London, v. 402, p. 139, 1999.

CAIN, M.; MILLIGAN, B.G.; STRAND, A.E. Long distance seed dispersal in plant populations American Journal of Botany, v. 87, n. 9, p. 1217-1227. 2000.

CLARK, D.A.; CLARK D.B. Spacing dynamics of a tropical rain forest tree: evaluation of the Janzen-Connell model. American Naturalist, v.124, p. 769-788, 1984.

CLARK, D A. La Selva: Ecology and Natural History of a Neotropical Rainforest. McDade L A, Bawa K S, Hes-penheide H A, Hartshorn G S., editors. Chicago: Univ. of Chicago Press, pp. 90-105, 1994.

CLARK, J. S., B. BECKAGE, P. CAMILL, B. CLEVE-LAND, J. HILLERISLAMBERS, J. LICHTER, J. MCLA-CHLAN, J. MOHAN \& P. WYCOFF. Interpreting recruitment limitation in forests. American Journal of Botany,

v.86, n. 1, p. 1-16, 1999.

CONNELL, J H. Dynamics of Populations. den Boer P J, Gradwell G R., editors. Wageningen, The Netherlands: Centre for Agricultural Publishing Documents, pp. 298-312, 1970.

CORDEIRO, N. J. \& H. F. HOWE. 2003. Forest fragmen-tation severs mutualism between seed dispersers and an endemic African tree. Proceedings of the Natio-nal Academy of Science, New York, v. 100, p. 14052-14056, 2003.

CRAWLEY, M J. Seeds: the Ecology of Regeneration in Plant Communities. Fenner M., editor. Wallingford: 
CAB, pp. 157-191, 1992.

DAILY, G. C. Ecological forecasts. Nature, London, n. 411 , p. 245,2001

DENSLOW, J S.; HARTSHORN, G S. Treefall gap environments and forest dynamic procesess. in McDADE, L.A.; BAWA, K.S.; HESPENHEIDE, H.A.; HARTSHORN, G.S. editors. McDADE, L.A.; BAWA. La Selva: Ecology and Natural History of a Neotropical Rainforest, p. 120-127, 1994.

DUNCAN, R. S. \& C. A. CHAPMAN. Limitations of animal seed dispersal for enhancing forest succession on degraded lands. In Levey D. J., W. R. Silva \& M. Galetti (eds.). Seed dispersal and frugivory: ecology, evolution and conservation. Wallingford, CABI Publishing, p. 437-450, 2002.

ESTRADA, A., R. COATES-ESTRADA, D. A. MERRIT JR., S. MONTIEL \& D. CURIEL. Patterns of frugivore species richness and abundance in forest islands and in agricultural habitats at Los Tuxtlas, Mexico. Vegetatio, v. 107, n. 108, p. 245-257, 1993.

GALETTI, M.; PIZO, M. A. Fruit eating by birds in a forest fragment in southeastern Brazil. Ararajuba, n. 4, p. 71-79, 1996.

GALETTI, M.; ALVES-COSTA, C. P.; CAZETTA, E. Effects of forest fragmentation, anthropogenic edges and fruit color on the consumption of ornithocoric fruits. Biological Conservation, Oxford, v. 111, n. 2, p. 269-273, June 2003.

GRAHAM, C. H. Factors influencing movement patterns of Keel-billed toucans in a fragmented tropical landscape in southern Mexico. Conservation Biology, Cambridge, v. 15 , n. 6, p. 1789-1798, Dec. 2001.

HARPER, J L. Population Biology of Plants. London: Academic Press, 892 p., 1977.

HERRERA, C.M.; JORDANO, P.; LOPES-SORIA, L.; AMAT , J.A. Recruitment of a mast-fruiting, bird-dispersed tree: bridging frugivore activity and seedling establishment Ecological Monographs, v. 64, p. 315-344.

HOWE, H. F.; SMALLWOOD, J. Ecology of seed dispersal. Annual Review of Ecology and Systematics, v. 13, p. 201-228, 1982.

HOWE, H. F. Implications of seed dispersal by animals for tropical reserve management. Biological Conservation , v. 30, p. 261-281, 1984.

HOWE, H. F.; SCHUPP, E. W.; WESTLEY, L. C. Early consequences of seed dispersal for a neotropical tree (Virola surinamensis). Ecology, Durham, v. 66, p. 781-791, 1985.

HOWE, H. F. Seed dispersal by fruit-eating birds and mammals. In: MURRAY, D. R. (Ed.). Seed dispersal.
Sydney: Academic Pr. Australia, p. 123-189, 1986.

HUBBELL, S P.; FOSTER, R B. Plant Ecology. Crawley M J., editor. Oxford: Blackwell; 1986. pp. 77-96.

JANZEN, D H. Herbivores and the number of tree species in tropical forest. American Naturalist, n.104, p. 501-528, 1970.

JANZEN, D. H.; MILLER, G. A.; HACKFORTH-JONES, J.; POND, C. M.; HOOPER, K.; JANOS, D. P. Two Costa Rican batgenerated seed shadows of Andira inermis (Leguminosae). Ecology, Durham, v. 57, p. 1068-1075, 1976.

JORDANO, P.; GODOY, J.A. Frugivore-generated seed shadows: a landscape view of demographic and genetic effects, In Levey, D. J., Silva, W. \& Galetti, M. (eds.). Frugivores and seed dispersal: ecological, evolutionary, and conservation. $C A B$ International, Wallingford, UK, p. 305-321, 2002.

JORDANO, P. Pre-dispersal biology of Pistacia lentiscus (Anacardiaceae): cumulative effects on seed removal by birds. Oikos, Lund, v. 55, p. 375-386, 1989.

JORDANO, P. Fruits and frugivory. In: FENNER, M. ed. Seeds: the ecology of regeneration in plant communities. Washington, C.A.B. International. p.105-156, 1992.

JORDANO, P., M. GALETTI, M.A: PIZO, and W .R. SILVA. Ligando Frugivoria e Dispersão de sementes à biologia da conservação. In: Duarte, C.F., Bergallo, H.G., Dos Santos, M.A., and V a, A.E. (eds.). Biologia da conservação: essências. Editorial Rima, São Paulo, Brasil, p. 411-436, 2006.

KATUSIC-MALMBORG, P.; WILLSON, M.F. Foraging ecology of avian frugivores and some consequences for seed dispersal in an Illinois woodlot. Condor, v. 90, p. 173-186, 1988.

KRIJGER C.L.; OPDAM, M.; THÉRY, M.; BONGERS, F. Courtship behaviour of manakins and seed bank composition in a French Guianan rain forest., Journal of Tropical Ecology, v. 13, p. 631-636, 1997.

KRUGEL, M.M.; BURGER, M.I.; ALVES, MARCO A. Bird frugivory on Nectandra megapotamica (Lauraceae) in an area of deciduous seasonal forest in Rio Grande do Sul, Brazil. Iheringia, Série Zoológica., Mar., v. 96, n.1, p.17-24, 2006. ISSN 0073-4721.

LAURANCE, W. F.; BIERREGAARD JUNIOR, R. O.; GASCON, C. Tropical forest fragmentation: synthesis of a diverse and dynamic discipline. Chicago: University of Chicago, 1997.

LAURANCE, W. F.; BIERREGAARD JUNIOR, R. O., editors, Tropical forest remnants: ecology, management, and conservation of fragmented communities. University of Chicago Press, Chicago. 
LEVEY, D J. Ecology, Durham, v. 69, p. 1076-1089, 1988.

LOISELLE, B.A.; Blake, J.G. Dispersal of Melastome seeds by fruit-eating birds of tropical forest understory. Ecology, Durham, v. 80, p. 330-336, 1999.

LOISELLE, B.A.; Blake, J.G. Potential consequences of extinction of frugivorous birds for shrubs of tropical wet forest. In Levey D. J., W. R. Silva \& M. Galetti (eds.). Seed dispersal and frugivory: ecology, evolution and conservation. Wallingford, CABI Publishing, p. 397-406, 2002.

MARTIN, T. E.; KARR, J. R. Temporal dynamics of neotropical birds with special reference to frugivores in second-growth woods. Wilson Bulletin, v. 98, p. 38-60, 1986.

McKEY, D. Coevolution of Plants and Animals. Gilbert $\mathrm{L} E$, Raven $\mathrm{P} \mathrm{H}$., editors. Austin: Univ. of Texas Press, pp. 159-191, 1975.

McDADE, L.A.; BAWA, K.S.; HESPENHEIDE, H.A.; HARTSHORN, G.S. editors. McDADE, L.A.; BAWA. Diversity of long-term flowering patterns. In La Selva. Ecology and natural history of a neotropical rain forest. Chicago: Univ. of Chicago Press, pp. 120-127, 1994.

METZGER, J. P. Tree functional group richness and landscape structure in a tropical fragmented landscape in SE Brazil. Ecological Applications, New York, v. 10, n. 4, p. 1147-1161, 2000.

MORELLATO, L.P.C.; LEITÃO-FILHO, H.F. Padrões de frutificação e dispersão na Serra do Japi. In História natural da Serra do Japi: ecologia e preservação de uma área florestal no Sudeste do Brasil (L.P.C. Morellato, org.). Editora da Unicamp/Fapesp, Campinas, p. 112-140, 1992.

MULLER-LANDAU, H.C.; WRIGHT, S.J.; CALDERÓN, O.; HUBELL, S.P.; FOSTER, R.B. Assessing recruitment limitation: concepts, methods and case-studies from a tropical forest. In Levey, D. J., W. R. Silva \& M. Galetti (eds.). Seed dispersal and frugivory: ecology, evolution and conservation. CAB International, Wallingford, UK, p. 35-53, 2002.

MURRAY, K.G. Avian seed dispersal of three Neotropical gap-dependent plants. Ecological Monographs, v. 58, p. 171-198, 1988.

OCHOA-GAONA, S.; GONZALEZ-ESPINOSA, M.; MEAVE, J. A.; SORANI-DALBON, V. Effect of forest fragmentation on the woody flora of the highlands of Chiapas, Mexico. Biodiversity and Conservation, London, v. 13, n. 5, p. 867-884, May 2004.

$\mathrm{PIZO}, \mathrm{M}$. A. Seed dispersal and predation in two populations of Cabralea canjerana (Meliaceae) in the Atlantic forest of southeastern Brazil. Journal of Tropical Eco- logy, New York, v. 13, p. 559-578, 1997.

PRICE, O. F.; WOINARSKI, J.C.Z.; ROBINSON, D. Very large area requirements for frugivorous birds in monsoon rainforests of the Northern Territory, Australia. Biological Conservation v. 91, p. 169-180, 1999.

RAMAN, T.R.S. Effects of landscape matrix and plantations on birds in tropical rainforest fragments of the Western Ghats, India. CERC Technical Report No. 9. Nature Conservation Foundation, Mysore, India. Reid, N. Ecology, v. 70, p. 137-145, 2004.

RENJIFO, L. M. Effect of natural and anthropogenic landscape matrices on the abundance of subandean bird species. Ecological Applications, Tempe, v. 11, n. 1, p. 14-31, 2001.

RESTREPO, C.; GÓMEZ, N.; HEREDIA, S. Anthropogenic edges, treefall gaps, and fruit-frugivore interactions in a neotropical montane Forest Ecology, Amsterdam, v. 80, n. 2, p. 668-685, 1999.

SANTOS, T.; TELLERIA, J.L. Influence of Forest fragmentation on seed consumption and dispersal of spanish juniper Juniperus thurifera. Biological Conservation, v. 70, p. 129-134, 1994.

SARGENT, S. Seed fate in a tropical mistletoe: The importance of host twig size. Functional Ecology. v. 9, p. 197-204, 1995.

SAUNDERS, D. A.; HOBBS, R. J. Nature conservation 2: the role of corridors. Chipping Norton: Surrey Beatty \& Sons Pty., 442 p., 1991. 